\title{
Editorial
}

\section{Biological fuel cells: Divergence of opinion}

Biofuel cell research has attracted considerable attention over the last decade, e.g., according to "The Web of Science" the number of publications per year in this area has been continuously increasing from 108 in 2004 to 1782 in 2014. In this short communication written in a provocative manner we, the guest editors of this Special Issue of Bioelectrochemistry devoted to biofuel cells, try to overstress the differences in current viewpoints in order to emphasize existing problems rather than just describe current opinion and praise to the skies perceived outstanding achievements, though these definitely exist in the field of bioelectrochemistry in general, and biological fuel cell research in particular. Emphasis on problems and disagreements can help push the field even further, resulting in a better understanding of the fundamental principles of the operation of biocatalysts. We do also believe that provoking the scientific community brings us much more closer to the final end point of all our research, i.e., general (large-scale) usage of "green" renewable biodevices for electric power generation and possibly even for concomitant storage of electric energy (vide infra).

\section{Possible classifications: influence on terms and definitions}

What is the exact definition of a biofuel cell? In a broad sense a biofuel cell is a device that converts chemical energy into electric energy using substances, which are defined as biofuels. It means that commercially available devices utilizing non-biogenic catalysts are biofuel cells, when operating on a biofuel, and that they are just fuel cells, when operating on a fuel. Within a narrow definition, which actually was used for this Special Issue, a biological fuel cell is a device that utilizes at least one biogenic catalyst in order to assimilate a fuel and an oxidant to generate electric power. In this narrow definition the origin of the fuel is less important. However, because of the somewhat generalized use of the term "biofuel cell" exploited for the two types of device, certain confusion does exist in the field. Very often one can find papers about "enzymatic biofuel cells" and sometimes "microbial biofuel cells", which should read "enzymatic fuel cells" or "microbial fuel cells", even though the authors of those reports do not even touch the field of biofuels. In other words, if in the term a biogenic catalyst is already used, in a broad form, like an enzyme or a microbe, or in a specific way, using the names of an enzyme or a microorganism, it is absolutely unnecessary and also very confusing to write "biofuel". One can use "biofuel cell" as a short abbreviation, when a biocatalyst(s) is not mentioned, since in this case "bio" means a biocatalyst but not a biofuel. There have been a large number of review papers on biofuel cells mainly focusing on the description of the current state of the art rather than focusing on classification and terminology. Only very recently, papers are emerging, in which terminology and classification of the biofuel cell are touched, see e.g., recent reviews in ChemPhysChem for enzymatic fuel cells (doi:10.1002/cphc.201300044) and in Energy \& Environmental Science (doi:10.1039/c4ee03359k) for microbial electrochemical systems. Moreover, most electrodes of modern biological fuel cells are based on biomodified nanomaterials or even complex nanobiocomposites with a huge double-layer and also pseudocapacitance. Indeed, recently hybrid biodevices based on double-function electrodes, i.e., electrodes with well-pronounced bioelectrocatalytic and capacitive features, were disclosed, which are able to generate and store electric power simultaneously. Should we call these hybrid devices "chargestoring biological fuel cells" or "self-charging biosupercapacitors", since they are electrochemical capacitors as well? To conclude, while general electrochemical concepts, terminology, and symbols are extensively described in the literature and well-recognized by the IUPAC (see Cohen at al., IUPAC Green Book, 3rd Edition, 2008), including electrochemical biosensors - widely used bioelectronic devices, another type of bioelectronics, biological fuel cells stay somehow in the shadow of IUPAC and yet this intensively developing field obviously calls for a unified approach and a terminology that is agreed on a worldwide basis.

\section{Measures: proper evaluation and comparison}

One of the main goals of the fundamental part of biofuel cell research in particular, and bioelectrochemistry in general, is to properly wire biocatalysts to electrode surfaces. "Properly" here refers to that neither activity nor stability of a biocatalyst should be significantly altered in a negative way. "Properly" also means that the heterogeneous electron transfer rate should be fast enough for operation of the biocatalyst in order to provide as high a bioelectrocatalytic current as possible. In order to understand the limiting factors of bioelectrocatalytic processes, detailed bioelectrochemical investigations should be carried out, since usually high performance biodevices operate in a complex mixed kinetic regime or they are even strictly diffusion limited. Moreover, in biological fuel cell research one should always remember Ohmic losses and other possible limitations, which should be taken into account when evaluating high performance biodevices. It means that just a comparison of current densities and operating voltages without a clear understanding is definitely not good enough!

\section{Current achievements: are we ahead from the 60s?}

Since the last two decades, microbial fuel cell research made a tremendous step forward with impressive fundamental and technological achievements. Indeed, practical usage of microbial devices has been realized already. However, enzymatic fuel cell research is stagnant somehow in spite of loud intriguing statements concerning the possible practical use of enzymatic fuel cells as electric power sources for self- 
contained bioelectronics operating in humans ex vivo and in vivo. Over the last few years researchers have started to focus on one of the major problems, which seems to hinder practical application of enzymatic devices, i.e., their limited operational stability. Indeed, a lot of investigations are going on to improve the operational stability of redox enzymes using modern scientific developments in the field of natural and induced mutagenesis. However, is the stability of redox enzymes really the main problem, the only problem, and is it actually a problem at all?

\section{Future perspectives: radiant versus obscured}

Not a single bioelectrochemist would wish to become the enemy of any microbiologist or enzymologist, since they constantly provide conventional and novel, wild type and specifically engineered bioelements, desperately needed in our research. Nevertheless, we would like to emphasize that limited operational stability is an intrinsic property of a biological object including oxidoreductases, of course. In our opinion, the question: "Will biologists be able to beat Nature designing extraordinarily robust and stable, but highly active and efficient oxidoreductases?" is very close, if not identical, to the question concerning unlimited life-time for a human. Certain parameters always need to be downplayed. There are impressive scientific achievements showing tremendous improvements in the operational stability of oxidoreductases. However, very often these improvements are compromised by a significant decrease in the efficiency of the catalytic performance of an enzyme. We do agree that it might be feasible today to design a robust protein globule stable for decades or even centuries, if other degradation factors, e.g., bacterial infection, are avoided. But will this extremely robust biomolecule be catalytically active at all? We might prolong our life-time to millions of years.... But will we be humans or "angels/devils" in that case? By no chance would we like to be recognized as mumbled nags of the field or, even worse, as scientific inquisitors killing off the most advanced and interesting, though challenging and risky, biological projects including specific engineering of biocatalysts with desired properties. We just want to emphasize the following question: "Would it also be clever to use already existing achievements in Nature developed over millions and millions of years of evolution?". In other words, would it be more realistic, at least at the current stage, to use high performance miniature, but relatively unstable, enzymatic fuel cells and intrinsically stable mascroscale microbial electrochemical systems for short-term and long-term applications, respectively, whereas organelle based fuel cells might occupy a niche somehow in between?

Last but not least, biological fuel cells are devices intended for electric power generation. We are very sorry for our somewhat childish reminder, but the electric power produced is either dissipated as heat or consumed by electric devices in diverse forms. During the last few decades low power/low voltage milli- and micro-scale electronics have made huge progress towards the nano- and even the pico-scale, when one talks about both size and power. As just one example, a fully functional system of only $1 \mathrm{~mm}^{3}$ size used for continuous pressure monitoring, which consumes only few tens of $\mathrm{nW}$ of eclectic power, has been recently reported by researchers and engineers from Michigan University (http://www.eecs.umich.edu/eecs/about/articles/2015/ Worlds-Smallest-Computer-Michigan-Micro-Mote.html). It is hard to believe, but this self-contained system incorporates a wireless communication unit, and an in-built computer, which are really microscale. In one simple phrase, one can say that what 100 years ago was just noise, 50 years ago had already become a signal. However, extremely small currents and voltages already represent quite a significant amount of electric power output nowadays. Nevertheless, very often bioelectrochemists do blame each other for low voltages and low currents of the biological fuel cells developed, with the strong statement that these are useless devices. We are not actually blamed by physicists or pure electrochemists, but it seems that we love to attack each other, possibly, due to the quite narrow field in which we are competing. In spite of tremendous progress in electronics, there is an opinion in the bioelectrochemical community that low current-low voltage biological fuel cells cannot power modern electronic gadgets, which require $2.5 \mathrm{~V}$ and higher. However, one should remember the basics of physics that $2.5 \mathrm{~V}$ is unnecessary for operation of even semiconductor based transistors: we just create such relatively high voltage gadgets because of certain operational reasons, e.g., to avoid unnecessary losses while still keeping the voltage quite low for security reasons. Today, there are commercially available transistors operating at $0.5 \mathrm{~V}$ and even lower. Furthermore, modern devices built on organic and inorganic materials do work on just a few $\mathrm{mV}$ of operating voltage. In the case of electric power consumption in the nW range even $0.1 \mathrm{~V}$ would be more than enough. Taking all this into account, researchers working in the field of biological fuel cells should never be blamed for $\mu \mathrm{W}$ power generation. Indeed, today this constitutes a huge amount of electric power, fully sufficient for the design of self-contained, i.e., self-powered and wireless, practically useful bioelectronic devices.

Sergey Shleev Biomedical Sciences, Health and Society, Malmö University, 20560 Malmö, Sweden

Corresponding author.

E-mail address: sergey.shleev@mah.se.

Alain Bergel

Laboratoire de Genie Chimique, CNRS, University of Toulouse (INPT), 31432 Toulouse, France

Lo Gorton

Analytical Chemistry/Biochemistry and Structural Biology, Lund University, PO Box 124, 22100 Lund, Sweden 